\title{
Tool Localization in 3D Ultrasound Images
}

\author{
Paul M. Novotny ${ }^{1}$, Jeremy W. Cannon ${ }^{1,2}$, and Robert D. Howe ${ }^{1}$ \\ ${ }^{1}$ Division of Engineering and Applied Sciences, Harvard University, Cambridge, MA \\ novotny@fas.harvard.edu \\ http://biorobotics.harvard.edu \\ ${ }^{2}$ Department of Cardiac Surgery, Children's Hospital Boston, MA
}

\section{Introduction}

Real-time three-dimensional ultrasound has been demonstrated as a viable tool for guiding surgical procedures [1]. This visualization technique may enable a range of new minimally invasive techniques in cardiac and fetal surgery. It is becoming increasingly clear, however, that instruments such as endoscopic graspers when immersed in liquids display artifacts and irregularities that make it difficult to determine the tool's location, orientation, and geometry (Figure 1a). In addition, the instrument's shape can appear incomplete due to its orientation or obstacles in the field. Since the geometry and properties of these instruments are known a priori, it is feasible to combine this information with the ultrasound image data to locate and render an enhanced representation of the instrument. This paper introduces a preliminary study of enhancing tool display in three-dimensional ultrasound, focusing on the relationship between the tissue and instrument.

\section{Methods and Materials}

Images were acquired with a real-time three-dimensional ultrasound system (Live 3D Echo, Philips Medical Systems, Andover, Mass.) that produces 128x160x64 volumetric data at 20-25 volumes per second. The probe was placed at the surface of a water-filled tank containing a flat bovine tissue sample (flank steak). A $6.2 \mathrm{~mm}$ diameter acetal cylindrical rod was incrementally moved toward the tissue sample with a linear micrometer drive as images were recorded.

For instrument identification, the 3-D volume was first segmented using thresholding and subsequently divided into discrete candidate volumes by connecting neighboring voxels. Principle component analysis was performed on each candidate to find its direction of maximum variance [2]. The longest and straightest candidate (largest ratio of first and second principle components) was selected as the likeliest candidate for the rod. In addition, the direction of maximum variance was assigned to the major axis of the cylinder, defining its orientation. To find the position of the rod, the selected candidate's data points are projected onto a plane perpendicular to its axis (found earlier by principle component analysis). The center is calculated from a linear least squares fit of the projected points to a circle of known radius. 


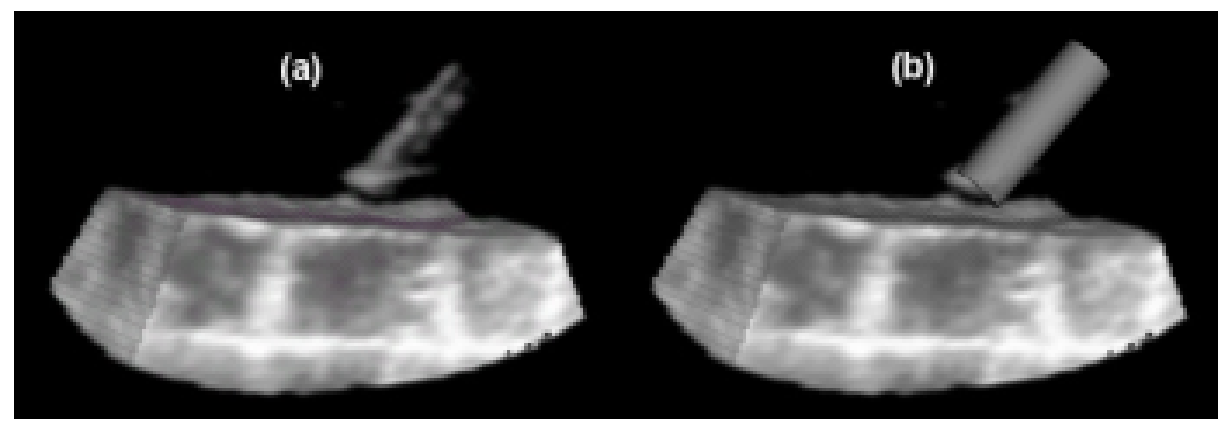

Fig. 1. 3D ultrasound images of a rod $2 \mathrm{~mm}$ from the tissue sample. (a) Raw image showing incomplete rod and artifacts; (b) Fitted tool overlay

With the orientation of the instrument's axis and a point along the axis, the location of the instrument tip is the only remaining parameter to fully constrain the fit. The tip is easily found by selecting the endpoint of the segmented point cluster along its principle axis.

\section{Results}

To verify our technique, the 3D ultrasound image was overlaid with a geometric representation of the cylinder at the calculated position and orientation. As can be seen in Figure 1, the overlaid rod accurately matches the ultrasound image of a rod $2 \mathrm{~mm}$ from the surface of the tissue sample. In addition, the overlay enhances the image by filling in missing structures, most notably the underside of the rod which is shadowed by the top of the rod.

It was found through numerous samples and positions that the orientation consistently matched well. However, the position of the tip did produce significant error (mean $0.7 \mathrm{~mm}$, standard deviation $0.6 \mathrm{~mm}$ ), largely as a consequence of the simple technique used to find the tip. Further study is under way to improve the accuracy of tip identification. In addition, future work will focus on more complex tool geometries in tank studies and in vivo.

\section{References}

1. Cannon, J., Stoll, J., Howe, R., Salgo, I., Knowles, H., Dupon,t P., Marx, G., and del Nido, P.: Real Time 3 Dimensional Ultrasound for Guiding Surgical Tasks. Computer Aided Surgery 2003, http://biorobotics.harvard.edu/pubs/Cannon_3DUSSurgery03.pdf; In Press

2. Draper, K. J., Blake, C. C, Gowman, L., Downey, D. B., Fenster, A.: An algorithm for automatic needle localization in ultrasound-guided breast biopsies. Medical Physics 27(2000) 1971-9 